\title{
El ser ahí de la vulnerabilidad y la potencia política de la eticidad performativa. Una lectura de Judith Butler en clave hegeliana
}

\author{
The Thereness of Vulnerability and the Political Scope \\ of Performative Ethical Life. A Reading of Judith Butler in \\ Hegelian Key
}

NURIA SÁNCHEZ MADRID*

\begin{abstract}
Resumen: Este ensayo pretende enfocar el trasfondo hegeliano clave para entender algunas posiciones de Judith Butler acerca de la subjetividad y el derecho universal a la ciudadanía, discutiendo algunos de sus escritos más recientes relacionados con manifestaciones y concentraciones populares y la ocupación del espacio público. Me ocuparé en primer lugar del intento de Butler de esbozar un nuevo marco de reconocimiento en clave hegeliana, capaz de denunciar toda violencia ética y de fundar algo así como una "vida ética performativa". En segundo lugar, me centraré en la conexión entre la identidad narrativa del yo y la estética del ser ahí [thereness] delimitada por la teoría de la performatividad política de Butler. Por último, me referiré a la reivindicación de esta autora de la existencia de una comunidad corporal "no elegida" y "pre-contractual" que desafía a la política denunciando la producción neoliberal de
\end{abstract}

\begin{abstract}
This essay mainly attempts to highlight the Hegelian background of Judith Butler's key assertions on human identity and the universal right to citizenship, by discussing most of her more recent writings regarding popular demonstrations and occupation of the public space. I shall tackle first Butler's project to sketch a new frame of recognition in Hegelian frame, able to denounce and dissolve ethical violence and to ground what I suggest to name as "performative ethical life". Second, I will focus on the connection between the narrative identity of self and the aesthetics of thereness shaped by Butler's theory of political performativity. Finally, I will address Butler's claim of an "unchosen" and "pre-contractual" bodily community that challenges politics to denounce the neoliberal production of precarity, rejecting the declaration of human beings as non-human.
\end{abstract}

Fecha de recepción: 24/07/2016. Fecha de aceptación: 15/09/2016.

* Profesora Contratada Doctora de la Facultad de Filosofía de la UCM. Dirección de contacto: nuriasma@ ucm.es Sus líneas de trabajo están relacionadas con la Historia de las Ideas Políticas, la Filosofía Antigua y Contemporánea y la Antropología filosófica. Recientes publicaciones suyas, cuya edición también ha tenido a su cargo, son: (en colaboración con A. Pinzani y A. Faggion), Kant and Social Policies, Palgrave McMillan, 2016; (con Larry Krasnoff y P. Satne), Kant's Theory of Right in the XXIth Century, Univ. of Wales Press, 2016 y Hannah Arendt y la literatura, Barcelona, Bellaterra, 2016. Este trabajo procede de una investigación resultante de los Proyectos Naturaleza humana y comunidad (III). ¿Actualidad del humanismo e inactualidad del hombre? (FFI2013-46815-P) y Retóricas del Clasicismo. Los puntos de vista (contextos, premisas, mentalidades) (FFI2013-41410-P), concedidos ambos por el MINECO del Gobierno de España. 
la precariedad y rechazando la desatención a la humanidad y dignidad de todos los seres humanos. Palabras clave: Judith Butler, eticidad performativa, vulnerabilidad, precariedad, democracia radical.
Keywords: Judith Butler, Performative Ethical Life, Vulnerability, Precarity, Radical Democracy.

\section{La vida ética performativa según Butler}

Judith Butler ha elogiado con frecuencia la perspicacia de Hegel para detectar la capacidad de un sistema ético para transformar radicalmente la lucha por la vida y la muerte que origina la forma política estatal. ${ }^{1}$ Con todo, debe atenderse suficientemente al hecho de que Butler sustituye la atracción de Hegel por la vida institucional por el cuidado de la visibilidad y precariedad del cuerpo, al que considera en condiciones de percibir y aprehender los defectos de reconocimiento de nuestro tiempo. «Aprehensión puede convertirse en la base para una crítica de normas de reconocimiento», sostiene Butler en Frames of war ${ }^{2}$, subrayando los límites de lo que es reconocido normalmente como meramente humano. Es más, esos límites deberían revisarse con toda atención, puesto que podrían abrirse con ello nuevas vías de emancipación. La aprehensión ayuda a entender el mundo desde una perspectiva más amplia que la que proporciona el reconocimiento social, por lo que esta modalidad de juicio ha de ser fomentada y cultivada según Butler. Cuando el sujeto gana la posibilidad de aprehender las limitaciones de su propio marco de reconocimiento emerge un nuevo modelo post-hegeliano de reconocimiento, para el que la opacidad parcial y la ceguera que cada ser humano comparte con otros suministra otro sentido de la identidad, ya no sujeta al ideal de una auto-identidad continuamente idéntica a sí misma. ${ }^{3}$ Esta identidad porosa, "deshecha" por la influencia de la exterioridad, está rodeada por una "temporalidad social que excede sus propias capacidades para la narración" ", lo que destaca lo limitado del marco de partida. Naturalmente, será complejo exponer las condiciones del propio discurso a través de precisamente el propio discurso, pero reconocer las interrupciones e incluso las fallas de una pretendida propiedad del sí mismo proporcionará al sujeto la oportunidad de ser crítico con las fronteras de su propia facultad de reconocimiento. Butler acostumbra aludir en este contexto a la condición que Adorno impone al cuestionar moral, a saber, el juicio que el sujeto proclama acerca del valor pretendidamente colectivo y universal de exigencias concretas. En realidad, la aproximación de Adorno a la violencia ética inspira la percepción de Butler sobre la presión social que ideas ya periclitadas siguen intentando ejercer sobre una sociedad, como confirma en Giving an account of oneself:

[El] ethos se vuelve violencia solo cuando se ha vuelto un anacronismo. Lo que resulta extraño históricamente -y temporalmente- en esta forma de violencia ética es que a pesar de que el ethos colectivo se ha vuelto anacrónico, no se transforma en

1 Butler, $\operatorname{GAO}(2005,29)$. Vd. también Hegel, Enciclopedia, § 433.

2 Butler, $F W(2009,5)$.

3 Butler, $G A O(2005,41-42)$.

4 Butler, $G A O(2005,7)$. Cfr. Ong-Van-Cung $(2010,138)$. 
pasado, sino que insiste en el presente como un anacronismo. El ethos rechaza convertirse en pasado y la violencia es la forma en que se impone al presente. En efecto, no solo se impone al presente, sino que intenta eclipsarlo -y este es precisamente uno de sus efectos violentos..$^{5}$

El pasaje es clarificador acerca de la destrucción que costumbres e ideas periclitados pueden suponer para el espacio público. Cuando las reglas éticas rechazan reconocer su hechura histórica y se resisten a disolver su autoridad, dejan de ofrecer un marco existencial amplio para la vida humana, desatando una violencia poderosa sobre la sociedad. Ahora bien, la percepción de puntos débiles en las normas éticas de una comunidad dará paso a una experiencia más fructífera de lo que la autonomía implica, apartando al "yo" de la idea de una posesión fija:

[T] enemos que reconocer que la ética exige de nosotros que nos arriesguemos precisamente en los momentos de incertidumbre, cuando lo que nos forma diverge de lo que yace fuera de nosotros, cuando nuestra disposición a deshacernos en relación con otros constituye nuestra oportunidad de convertirnos en humanos. Ser deshechos por otro es una necesidad primaria, una angustia, para ser del todo precisos, pero por eso mismo una oportunidad - de ser interpelados, exigidos, vinculados a lo que no es yo, pero también para ser movidos, urgidos a la acción, ser interpelados por otro lugar, y de esa manera vaciar al "yo" autosuficiente como una especie de posesión. Si hablamos e intentamos dar razón desde este lugar, no seremos irresponsables o, si lo somos, seremos seguramente perdonados. ${ }^{6}$

La ambivalencia entre actividad y pasividad aparece como un medio potente frente a la violencia ética y permite considerar nuestra vida como ontológicamente precaria, haciendo de la performatividad el destino de la condición humana. En efecto, precariedad, considerada no como una dimensión ontológica, sino como un producto social, condena a gente plural -mujeres, queers, transgénero, pobres o apátridas- a renunciar al derecho a ser reconocidos, legibles o llorados. Así, pues, esta experiencia de la falta inspira una acción performativa, como un proceso «que implica ser actuado en maneras que uno no siempre entiende completamente y de actuar consiguientemente de forma política». ${ }^{7}$ De esa manera, la gente que políticas concretas destina a la exclusión y al sometimiento comienza a experimentar su libertad en la medida en que la ejerce, demandando así ser vistos como un sujeto reconocible. Butler comparte con Adorno la impresión de que ser capaz de denunciar lo inhumano representa la contribución más efectiva para defender la dignidad humana ${ }^{8}$. Es más, considera que esta capacidad para denunciar debe mantener permanentemente abierta la noción de lo que debería ser reconocido como humano. Subraya esta apertura como «la paradoja

5 Butler, $G A O(2005,5)$. Todas las traducciones de los escritos de Butler son mías. Cfr. Adorno $(2000,17)$.

6 Butler, $G A O(2005,136)$.

7 Butler (2014b) «Performativity, precarity and sexual politics», vd. especialmente pp. vi-vii and xii-xiii: http:// www.aibr.org/antropologia/04v03/criticos/040301b.pdf (acceso el 11 de abril de 2015).

8 Vd. Adorno, $P M P(2000,175)$. 
permanente ${ }^{9}$ de la metafísica de Hegel, que no limita al Absoluto a desempeñar la función de un elemento que será infinitamente subsumido bajo una estructura sistemática, sino más bien la función de una transformación continua que no conoce finalidad alguna, stasis o cierre. ${ }^{10}$ Naturalmente, Hegel identifica al deseo con la búsqueda de reconocimiento, estableciendo un vínculo fuerte que ha de ser criticado radicalmente. Como la verdad del deseo, si resultara deseable hablar en estos términos, nunca se muestra del todo y el deseo mismo hace del sujeto un ente ficticio ${ }^{11}$, Butler sostiene que sería más prudente dejar la definición de "lo humano" abierta a formulaciones futuras, en condiciones de ampliar su campo de acción y susceptibles de incorporar progresivamente ulteriores cualidades y facultades. ${ }^{12} \mathrm{~A}$ juicio de Butler hay buenas razones para dejar "lo humano" sin clausurar y la mayor parte de ellas proceden de la retórica. De hecho, la definición acostumbra conferir una cualidad a una serie de individuos, arrebatando a otros la posibilidad de alcanzar ese estatus, «produciendo un diferencial entre lo humano y lo menos-que-humano $»^{13}$, un double bind que Butler intenta desentrañar, sustituyéndolo por la demanda performativa de derechos. Tal cambio en la percepción de uno mismo y de los otros garantizará el valor futuro de lo universal, en la medida en que comienza a atender a lo que Butler denomina «el límite contingente de la universalización», a saber, la Otredad que la constituye de manera esencial. ${ }^{14}$ Esta valoración de los términos provistos de un alcance universal en política y en ética comparte la crítica de la universalidad abstracta que Deleuze dirigió a los discursos institucionales sobre los derechos humanos, toda vez que Butler defiende también la prioridad de la performance para obtener ganancias jurídicas de manera exitosa. ${ }^{15}$ Esta consideración del valor de lo universal conduce a releer la historia de la Modernidad, con el fin de deconstruir los contextos de opresión e inequidad que sirvieron como escenario para moldear muchos conceptos políticos y éticos fundamentales. En esa medida, los conceptos siguen estando en condiciones de recibir significados desconocidos hasta el momento, tras haber arrojado luz sobre su conexión con una situación histórica concreta. Dicho de otra manera, esta tesis teórica sobre la universalidad política iniciaría un combate para ganar el derecho a universalizar todo término ético y político, desde el momento que conceptos clave no estarían nunca asegurados de antemano, manifestando así un compromiso con la "política del desconfort" acuñada por Foucault, en cuyos parámetros Butler también se reconoce. ${ }^{16}$

Ser consciente del marco social de los cuerpos facilitará la localización del modo y el tiempo en que la precariedad emerge, un proceso que debe ser exhaustivamente denunciado, por cuanto impide consumar los fines de la universalización. ${ }^{17}$ Las medidas para proteger la vida humana que produce simultáneamente la exclusión de otras vidas consideradas

9 Butler, $S D(1999,13)$.

10 Ibid.

11 Butler, $S D(1999,238)$.

12 Butler, $U G(2004,222)$.

13 Butler, $U G(2004,2)$.

14 Butler, $U G(2004,191)$.

15 Vd. "Human Rights" in Deleuze, http://www.generation-online.org/p/fpdeleuze10.htm (acceso el 15 de abril de 2015).

16 Butler, ES (1997, 160-161).

17 Butler, FW (2007, 3); cfr. I. Lorey (2011) «Governmental Precarization»: http://eipcp.net/transversal/0811/ lorey/en (acceso el 16 de abril de 2015). 
como amenazadoras es una falla penosa de muchas democracias liberales, «de suerte que la guerra puede ser enarbolada justificadamente en nombre de algunas vidas, mientras que la destrucción de otras vidas puede ser defendida también justificadamente». ${ }^{18}$ Dicho brevemente, Butler rechaza aceptar la lógica de la exclusión, sustituyéndola por una exigencia performativa de equidad universal.

\section{La narratividad del ser ahí [thereness] y la emergencia del pueblo}

Cuando la vida humana observa que sus derechos básicos son fulminados por un poder presuntamente legítimo o por uno impuesto salvajemente, Butler anima a hacer de la performatividad un instrumento válido para alcanzar el objetivo de la emancipación. Asambleas y manifestaciones populares parecen desempeñar en tales situaciones la función que Kant calificó de "signo histórico" de un progreso humano en la historia, esto es, la posibilidad de dar un giro a la política con vistas a objetivos emancipatorios. En efecto, las manifestaciones populares amplían el espacio en que los seres humanos aparecen y construyen el llamado espacio público, desde el momento que el ultimo no es un fenómeno siempre a la mano, sino un bien común construido y conservado por el trabajo humano. Esta tesis obedece a la crítica que Butler dirige a la displicencia que Hannah Arendt siempre mostró por las condiciones materiales del espacio público, que Butler considera como una estructura básica de acción y discusión. Trataré en la tercera sección acerca de las dimensiones materiales del espacio de aparición que han de ser contempladas para entender el sentido cabal de la publicidad de acuerdo con esta teoría. Pero ahora me concentraré en lo que Butler analiza como acciones corporales que aspiran a ampliar el alcance de normas, leyes y reglas, subrayando -como ella misma hace- la enigmática rebelión de una ni niña ni mujer, como es Antígona, cuya resistencia a aceptar su exclusión social conmociona las jerarquías de la polis ${ }^{19}$. La gente excluida es forzada a exigir un lugar en el mundo a través de su propia acción, sin pedir previamente permiso para ocuparlo. Dicho de otra manera, Antígona osa expresarse por medio de sus propios movimientos y acciones, con lo que consigue ampliar las categorías aceptadas por la polis, haciendo de la catacresis política un instrumento para promover lo "menos que humano" al estatuto de la dignidad humana. De esa manera, Antígona confirma que la ocupación del espacio precede al reconocimiento de derechos, dado que consigue que roles y demandas sociales reciban inteligibilidad social que el gobernante de la polis percibe como causa de su propia supresión. Esta hostilidad está basada en la capacidad de seres discursivos pretendidamente no-humanos para jugar con las fronteras del parentesco y la ciudadanía, como condiciones que moldean la identidad humana. Butler señala especialmente la presencia potencial que la existencia corporal humana es capaz de desempeñar en público, pues esta modalidad de existencia fomenta una experiencia de la ley que por primera vez somete la normatividad a las necesidades y deseos de los cuerpos humanos. Así, podría decirse que la corporalidad del derecho es una dimensión esencial para este análisis de la universalidad política, desde el momento que el cuerpo es el primer elemento que ha de exigir respeto para su espacio propio y circunstancias en el ámbito compartido por otros

18 Butler, $F W(2007,53)$.

19 Butler, $A C(2000,82)$. 
seres humanos. Naturalmente, el cuerpo es un asunto ambiguo en el pensamiento de Butler, pero su ambivalencia despliega tanto una capacidad asombrosa para ser herido, explotado o castigado, como, por el contrario, una sólida resistencia frente a comportamientos intolerables. Butler argumenta a favor de esta ambigüedad positiva del cuerpo humano:

Me parece que hay una presencia implicada por la idea de exposición corporal que puede convertirse en ocasión tanto de subyugación como de reconocimiento. La exposición forzada de los cuerpos en los checkpoints y otros lugares de vigilancia intensiva puede ser un ejemplo de lo anterior. El cuerpo tiene que comparecer, moverse únicamente de acuerdo con el movimiento y velocidad exigidos por el soldado o la máquina (o el soldado-máquina híbrido). Podemos decir que en esos ejemplos la persona que tiene que pasar por los checkpoints está "presente" de una manera que está ligada al sometimiento. Pero de manera similar, cuando actos de resistencia ocurren en un checkpoint, cuando los cuerpos aparecen o se mueven de maneras que no están autorizadas, o cuando comunidades se forman con vistas a limitar o resistir prácticas militares, un cierto tipo de presencia tiene lugar ${ }^{20}$.

El texto vuelve al dictum de Spinoza "nadie sabe lo que puede un cuerpo", por cuanto subraya el poder de resistencia que los seres humanos ponen de manifiesto cuando están controlados por una autoridad institucional o por una fuerza arbitraria. A pesar de que la exposición y de la debilidad parece caracterizar a este tipo de fenómeno social, el "guión” de esta sujeción no puede evitar que los agentes sometidos se rebelen contra tales situaciones, intentando romper con el ritual de control, que queda reemplazado por la exhibición de la autonomía humana. Así, el cuerpo humano que rechaza abandonar el lugar que se le ha asignado y exige el derecho a su propio lugar, demuestra que las acciones capaces de oponerse al poder militar o disciplinario pueden jugar más de lo previsto con las órdenes dirigidas al cuerpo. Otro ejemplo habitual procede de los manifestantes de Baltimore durante los disturbios raciales de 2015, que tras el ultimo caso de violencia ejercida por un policía gritaron que "la vida negra importa", usando su propio cuerpo como un instrumento de resistencia física.

Butler sostiene que las reglas están destinadas a ser subvertidas precisamente por los seres humanos que gobiernan con ellas y que la subversión promueve una modalidad de libertad latente en el campo del sometimiento. Se trata de un mecanismo psíquico que Butler ha trabajado intensamente en The Psychic Life of Power. Este análisis de la manifestación política de los cuerpos humanos también remite a los sujetos heroicos que rechazaron ser tratados como seres no-humanos o como seres humanos de segunda o tercera categoría, afirmando su derecho a la justificación y la normatividad. En un libro reciente, escrito junto con Athina Athanasiou, Butler recuerda la proeza de Rose Parks cuando rechazó entregar su asiento en un autobús a los pasajeros blancos a los que correspondía sentarse en las primeras filas. El quid de la acción obedece a que Parks decidió un día pensar y actuar con la ayuda de su propio cuerpo, haciendo de sí misma un "escudo humano" frente a la humillación y denigración. En efecto, obtuvo un notable éxito con esta acción espontánea, que fue realizada no desde el punto de vista de una autonomía poseída, sino desde la intención de alcanzarla.

20 Butler/Athanasiou (2013, 13-14). 
Butler concede un significado especial a las propias palabras de Parks, cuando esta señaló en su autobiografía que en realidad no fueron ni la fatiga ni una edad avanzada los motivos que despertaron en ella tal reacción. Más bien fue el hartazgo producido por la falta de agencia -el cansancio producido por haber cedido constantemente- lo que le transmitió el coraje para permanecer tozudamente en su asiento contra las reglas establecidas. ${ }^{21}$ La historia de Rose Parks revela un hecho que Butler trata en otros escritos, a saber, la profunda ambivalencia de la vulnerabilidad humana, una condición que simultáneamente expone a los seres humanos a abusos y les dispone a rebelarse contra tales prácticas de sujeción. En realidad -afirma Butler- el fundamento de la ambivalencia tiene que rastrearse hasta sus últimas bases ontológicas, que subyacen a todos nuestros conceptos políticos. Tal análisis genealógico modificará la concepción usual de la vulnerabilidad, por cuanto mostrará el rostro jánico de esta y subrayará su agencia política ${ }^{22}$.

Esta aproximación a la condición vulnerable de los seres humanos podría ser considerada como una apología de la autodestrucción, como la misma Butler pone de relieve, si bien obviamente no es el caso en su valoración de las acciones descritas, ejemplificadas especialmente por los escudos humanos en medio de una guerra y por gente desarmada ante policías abusivos, en la medida en que sus comportamientos abren paso a procesos emancipatorios. De acuerdo con este punto de vista sobre la agencia humana, la aceptación de la vulnerabilidad genera un nuevo modelo de sujeto político, que procede del esfuerzo por superar situaciones de precariedad para construir una vida política. Así, la misma precariedad que debilita a los seres humanos les urge a poner fin a esta situación por medio de su propia acción. Como Butler ha señalado en varios textos ${ }^{23}$, la gente comienza a importar tan pronto como deciden ocupar un espacio y exigir allí su derecho a la existencia, denunciando una debilidad política que debería ser suprimida. De esta manera, la performatividad consigue fundamentar un nuevo orden ético, por cuanto promueve que personas pretendidamente excluidas o ilegales se vuelvan capaces de realizar una vida política, obteniendo con ello dignidad y reconocimiento como seres humanos. Nada de ello sería posible sin lo que sugiero denominar "estética del ser ahî", que actualiza el potencial político replegado en todo ser humano y manifestado cuando la gente se junta con fines reivindicativos en las calles, los parques y otros lugares públicos. Cuando la gente se manifiesta y ocupa un lugar para proclamar sus demandas y quejas, descubren que son un cuerpo político, que resiste para no ser desprovisto de representación y que habla con una voz plural. Lejos de aceptar la desposesión, esta gente, reunida y acostumbrada a soportar dificultades materiales, rechaza seguir siendo la cara oculta de la vida política institucional, decidiendo cómo desean ser representados y qué entienden por equidad. ${ }^{24}$ De esta manera, captan la importancia de estar en condiciones de espaciar la aparición, en lugar de aguardar pacientemente hasta recibirla

21 Butler/Athanasiou (2013, 21-22).

22 Butler (2014b) «Rethinking Vulnerability and Resistance»: http://www.institutofranklin.net/sites/default/files/files/Rethinking\%20Vulnerability $\% 20$ and $\% 20$ Resistance\%20Judith\%20Butler.pdf) (acceso el 15 de abril de 2016).

23 Butler/Athanasiou $(2013,101)$.

24 Butler (2011) «Bodies in alliance and the politics of the street»: http://www.eipcp.net/transversal/1011/butler/ en) (acceso el 10 de abril de 2015). 
de manos ajenas como un regalo. ${ }^{25}$ Así, defienden uno de los presupuestos básicos de la democracia, a saber, el deber de las instituciones políticas públicas de representar al pueblo:

El "estamos aquí" que traduce que la presencia corporal colectiva podría ser releído como "Todavía seguimos aquí", significando algo así como: "Todavía no hemos sido desposeídos. No nos hemos deslizado tranquilamente por las sombras de la vida pública: no nos hemos convertido en la brillante ausencia que estructura vuestra vida pública". En cierta manera la asamblea colectiva de los cuerpos es un ejercicio de la voluntad pública, y una manera de afirmar en forma corporal uno de los presupuestos más básicos de la democracia, a saber, que las instituciones políticas y públicas están comprometidas con la tarea de representar al pueblo y, en esa misma medida, por vías que establecen la equidad como un presupuesto de la existencia social y política. ${ }^{26}$

Los ejemplos y argumentos mencionados prueban que Butler confía decididamente en una política de la presencia, desde el momento que la construcción de una comunidad material precaria permite que los seres humanos sean reconocidos como agentes políticos. De ese modo, Butler discrepa de la crítica que Arendt dirige a las metafísicas de la presencia en sus escritos sobre la acción, puesto que contempla la presencia corporal colectiva como un instrumento político más fuerte que la mayoría de los discursos políticos contemporáneos sobre los derechos humanos y la soberanía política. En esta misma línea, el cuerpo desempeña en Butler una función preeminente comparado con el discurso humano y esta evidencia conduce «hacia versiones alternativas de la universalidad que son tramadas por la misma labor de traducción». ${ }^{27}$ El lenguaje de una población reunida no debería ser confundido con o reducido al lenguaje de la autonomía y dominio. Más bien, tal lenguaje reconoce la continua labor de las traducciones y las transacciones como su propio destino. Por eso, la discusión sobre la estética del ser ahí autoriza extraer como conclusión que la lucha por el reconocimiento es siempre una dimensión contingente que debería tomar distancia con respecto a las constricciones de un proyecto identitario y redistribuir los términos prisioneros del discurso dominante. ${ }^{28}$ La lucha tozuda por el reconocimiento de la gente que ocupa el espacio público genera un fenómeno político que comporta una universalidad inclusiva y contribuye a moldear la identidad personal de los sujetos implicados en esta acción colectiva.

\section{Conclusiones. Las exigencias de la comunidad corporal}

La emancipación no puede ser planteada como un objetivo solitario en Butler, sino que está unida a una multitud de hechos que requieren la participación de la gente. Butler lo constata sin titubeo alguno: «la revolución ocurrió porque todos rechazaron volver a casa, permaneciendo enclavados en el suelo, actuando concertadamente». ${ }^{29}$ Por ello, las reivin-

25 Butler/Athanasiou $(2013,194)$.

26 Butler/Athanasiou $(2013,197)$.

27 Butler/Laclau/Žižek (2000, 179).

28 Butler/Laclau/Žižek $(2000,168)$.

29 Butler (2011) «Bodies in alliance and the politics of the street»: http://www.eipcp.net/transversal/1011/butler/ en) (acceso el 10 de abril de 2015). 
dicaciones populares no podrían entenderse sin hacer antes de la casa del pueblo, donde el "yo" descubre que él es también "nosotros" y así que una condición plural no elegida precede y provee a todas sus acciones una base sólida. Incluso cuando la precariedad marca su sello sobre los cuerpos, Butler afirma que también fomenta que una comunidad inesperada aparezca mediante una «interdependencia precontractual» que guía la búsqueda de valores en pos de la defensa de una vida equitativa:

Si hay un 'nosotros' que se reúne en asamblea allí, en un espacio y tiempo precisos, hay también un 'nosotros' que se forma a través de los media que convocan a la manifestación y retransmiten los acontecimientos, de modo que algún conjunto de conexiones globales están siendo articuladas, un sentido diferente del global procedente del 'mercado globalizado'. Y algún conjunto de valores está siendo actualizado en forma de una resistencia colectiva: la defensa de nuestra precariedad colectiva y persistencia en la producción de equidad y los modos, discursivos o no, de rechazar el transformarse en algo disponible. Cuando esto ocurre, actuamos desde un sentido de la precariedad contra un sentido de la precariedad y en coalición, con frecuencia en proximidades no elegidas, donde una interdependencia pre-contractual está en ejercicio -sentida a veces como alivio o exaltación, pero a menudo más bien como incomodidad, conflicto, algo apenas vivible. Pero es precisamente en coalición donde las condiciones de lo vivible se negocian en un tipo de resistencia que episódicamente ejemplifica y repetidamente demanda otro modo de coexistencia, uno que intenta esbozar la reivindicación equitativa de una vida vivible. ${ }^{30}$

Mi principal intención en este texto ha sido la de señalar que la experiencia de pertenencia a una comunidad define el sentido del sujeto con arreglo a los escritos que Judith Butler ha dedicado a la reflexión sobre la identidad. Así, la performatividad parte de una precariedad que la comunidad corporal percibe como una falla insoportable y regula la experiencia compartida de pertenecer a un orden ético común, que el sujeto no percibe como un producto social ya construido antes de él, puesto que participa de su misma génesis. En esta línea, Butler anima a considerar el rendir cuentas a propósito de uno mismo como un corolario de su fundamentación ontológica de la acción. La acción se adapta a un marco concertado que no está basado en una voz inequívoca, sino que más bien acepta discursos plurales, que comportan serias consecuencias para la historia que cada agente pretende tejer. ${ }^{31}$ Formulado de manera resumida, nadie está en condiciones de conocer cómo es realmente sin emprender la tarea de descubrir la comunidad a la que pertenece, una que no termina de construirse nunca del todo, sino que ha de ser continuamente reformulada a través de la lucha básica por conseguir el reconocimiento humano, social y político. ${ }^{32}$

30 Butler $(2012,195)$.

31 Con esta defensa de la pluralidad de voces implicadas en la discusión pública Butler se aproxima a las tesis recientemente defendidas por R. Forst (2013: 3).

32 Axel Honneth ha desarrollado una teoría del orden ético democrático [demokratische Sittlichkeit] que aspiro a poner en discusión con el orden ético performativo de Butler, una cuestión de la que me ocuparé en un próximo escrito. Sobre este asunto véase Honneth (2013, 119-126). 


\section{Bibliografía}

Adorno, T. (2000): Problems of Moral Philosophy (PMP), Stanford U.P., Palo Alto.

Butler, J. (2012): Parting ways. Jewishness and the critique of Zionism (PW), Columbia U.P.

Butler, J. (2009): Frames of War: When Is Life Grievable? (FW), Verso, New York.

Butler, J. (2005): Giving an Account of Oneself (GAO), Fordham University Press,.

Butler, J. (2004): Undoing Gender (UG), Routledge, London.

Butler, J. (2000): Antigone's Claim: Kinship between Life and Death (AC), Columbia U.P.

Butler, J. (1999): Subjects of desire. Hegelian reflections in XXth Century France (SD), Columbia U.P.

Butler, J. (1997): Excitable Speech: A Politics of the Performative (ES), Routledge, London.

Butler, J. (2014a): «Rethinking Vulnerability and Resistance»: http://www.institutofranklin. net/sites/default/files/files/Rethinking\%20Vulnerability $\% 20$ and $\% 20$ Resistance $\% 20$ Judith\%20Butler.pdf).

Butler, J. (2014b) «Performativity, precarity and sexual politics», vd. especialmente pp. vivii and xii-xiii: http://www.aibr.org/antropologia/04v03/criticos/040301b.pdf

Butler, J. (2011): «Bodies in alliance and the politics of the street»: http://www.eipcp.net/ transversal/1011/butler/en)

J. Butler/A. Athanasiou (2013): Dispossession. The performative in the political, Polity, NewYork.

Butler, J./Chakravorty Spivak, G. (2007): Who Sings the Nation-State?: Language, Politics, Belonging, Seagull Books.

Deleuze, G. (1996): http://www.generation-online.org/p/fpdeleuze10.htm From L'Abécédaire de Gilles Deleuze, avec Claire Parnet, Vidéo Éd. Montparnasse.

Forst, R. (2013): «Noumenal Power», Exzellenzcluster Normative Orders Working Paper, Univ. Frankfurt A.M.: http://publikationen.ub.uni-frankfurt.de/opus4/frontdoor/index/ index/docId/33217, pp. 1-24.

Forst, R. (2011): "The ground of critique: On the concept of human dignity in social orders of justification", en Philosophy and Social Criticism 3/9, pp. 965-976.

González Fisac, J. (2012): «Retórica y fenomenología. Exterioridad y vulnerabilidad del campo del lenguaje», en P. Soley-Beltrán/L. Sabsay (ed.), Judith Butler en disputa. Lecturas sobre la performatividad, Egales, Barcelona/Madrid, pp. 169-194.

Honneth, A. (2011): Das Recht der Freiheit. Grundriß einer demokratischen Sittlichkeit, Suhrkamp, Frankfurt a.M.

Lorey, L. (2011): «Governmental Precarization»: http://eipcp.net/transversal/0811/lorey/en

Ong-Van-Cung, S.K.(2010): «Reconnaissance et vulnerabilité, Honneth et Butler», Archives de Philosophie 43, pp. 119-141. 